\title{
Spindle Cell Sarcoma on a Rare Site
}

\author{
Zubda Malik ${ }^{1 *}$, Aziz Abdullah' and Tariq Siddiqi \\ ${ }^{1}$ Department of Urology, Liaquat National Hospital and Medical College, Karachi, Pakistan \\ ${ }^{2}$ Consultant Oncologist \& Hematologist, Dr. Ziauddin Hospital, Karachi, Pakistan
}

\begin{abstract}
Spindle cell sarcoma is a rare tumor. This study reports a case of male patient with large pelvic mass. Examination and MRI confirmed the presence of exophytic mass. Resection of the mass was done. Histopathology showed spindle cell sarcoma.
\end{abstract}

Keywords: Spindle cell, sarcoma, rare site.

\section{CASE PRESENTATION}

A case of 70 years old male, resident of Karachi, smoker presented in Urology OPD with complain of lower abdominal swelling since 4 months.

According to the patient, he noticed a swelling in the suprapubic area 4 months back which was initially small but it gradually increased in size. The swelling was associated with pain. There was no history of weight loss, bone pain or fever.

On examination, approximately $10 \times 8 \mathrm{~cm}$ mass was palpable in suprapubic area with cystic to solid consistency and irregular margins. Rest of systemic examination was unremarkable.

Trucut biopsy of the mass was performed on August $2^{\text {nd }}$, 2019 suggesting spindle cell sarcoma.

\section{RADIOLOGY DETAILS}

Patient had Magnetic Resonance Imaging (MRI) of pelvis with contrast done which showed a large exophytic mass in pubic region measuring about $6.6 \times 7.5 \times 5.2 \mathrm{~cm}$. The mass was compressing the root and shaft of the penis with intact fat planes with no abnormal signals within the root of the penis. There was no lymphadenopathy and no bony involvement.

\section{Further Management:}

Patient underwent excision of the mass on August $21^{\text {st }}$, 2019.

\section{Operative Findings:}

It showed solid cum cystic mass in the suprapubic region adherent to the cavernosal body. Both spermatic cords were adherent to the mass and were separated.

\section{HISTOPATHOLOGY DETAILS}

\section{Gross Examination:}

Specimen was received in formalin in one container. It

*Corresponding Author: Zubda Malik, Department of Urology, Liaquat National Hospital and Medical College, Karachi, Pakistan; Email: zubdamalik@hotmail.com

Received: December 31, 2019; Revised: June 04, 2020; Accepted: July 01, 2020 DOI: https://doi.org/10.37184/lntbj.2708-7808.2.7 consists of a single irregular firm partly ruptured mass measuring $11.5 \times 6 \times 6 \mathrm{~cm}$. Cut surface shows grayish brown necrotic and fleshy tumor measuring $11 \times 4.5 \times 4.1$ $\mathrm{cm}$. Representative sections were taken and submitted in 1 to 6 (LAZ).

\section{Microscopic Examination:}

Sections examined reveal a malignant neoplastic lesion composed of spindle shaped cells arranged in a vague pattern less to slightly fascicular pattern (Fig. 1). Intervening collagenous fibers are seen. The tumor cells exhibit moderate to marked nuclear atypia with scattered nuclei. Extensive areas of necrosis are noted. The tumor is infiltrating into the surrounding fat. The tumor is reaching the outer painted margin.

Immunohistochemical studies were performed by DAKO envision method using the following antibodies:

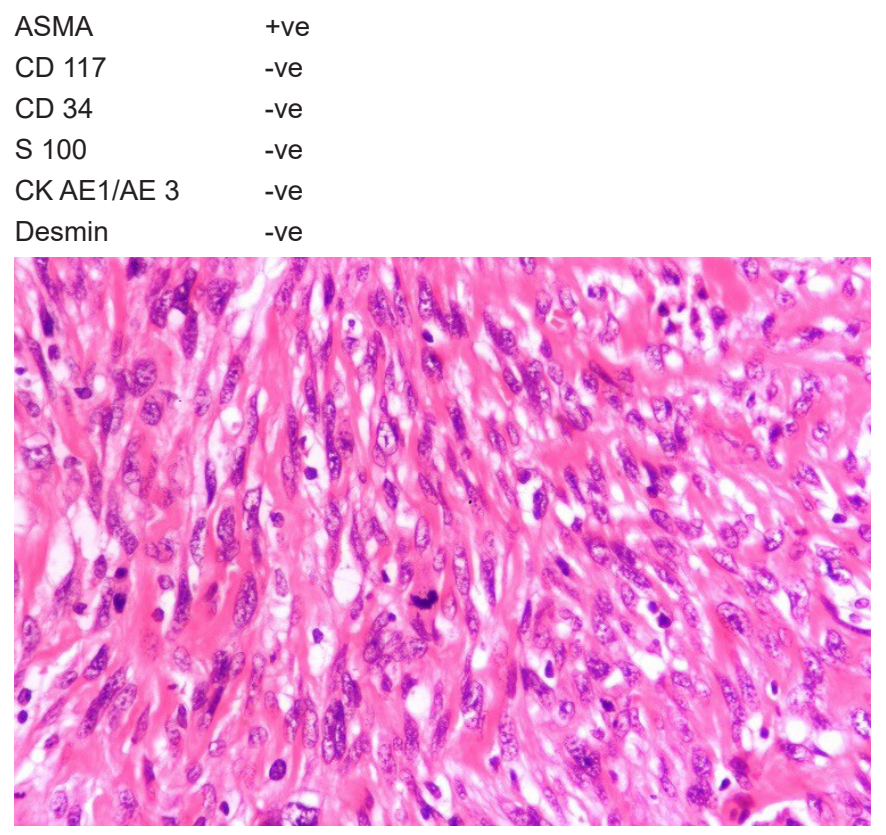

Fig. (1): Spindle cell sarcoma.

\section{Final Diagnosis:}

High Grade Sarcoma with Smooth Muscle Differentiation: The tumor is infiltrating into the surrounding fat and is 
reaching up to outer painted margin. Size of the tumor is $11 \times 4.5 \times 4 \mathrm{~cm}$.

\section{TUMOR BOARD DISCUSSION}

Participants included urologist, histopathologist, radiologist, oncologist and radiation oncologist.

How can this patient be managed further?

After the detailed case discussion among the participants, following options were suggested:

- Re-resection

- Adjuvant radiotherapy

- Resection followed by radiotherapy

What are the chances of negative surgical margin, if we go for re-resection?

Patient was counseled that in order to decrease the chances of positive surgical margins, we will have to acquire aggressive surgical approach which will include pubectomy and penile amputation. We discussed the possibility of reoccurrence with the patient and the family. However, patient did not agree for this aggressive approach so we had to go for palliative resection.

During the surgery, tumor was found to be adherent to the root of penis and posterior wall of pubic symphysis considering the wish of the patient, we limited our dissection and only shaved off the root of penis in order to get the maximum chances of negative surgical margin.

What are the indications of neoadjuvant radio therapy?

It is done when the surgery is difficult, in the cases of locally advanced disease as there are higher chances of positive surgical margins and post-operative reoccurrence. If getting clear margins is not possible at the moment then probably we have to go with radiation and then re-evaluate for surgery.

\section{CONCLUSION}

It has been decided in the tumor board meeting that patient will have repeat MRI to see the extent of residual disease. If based on repeat MRI, there is significant residual disease then the option is to reresect the tumor if clear margins can be obtained followed by adjuvant radiation therapy preferably IMRT vs neoadjuvant radiation therapy followed by resection of tumour.

\section{UROLOGIST'S PERSPECTIVE}

Spindle cell/sarcomatoid carcinomas are the rare variant of penile cancers and constitute 1-3\% with very poor prognosis and early vascular metastasis [1]. Majority of the patients had either regional (60-91\%) or distant metastasis at presentation or developed them on follow up and was associated with a high mortality (50-80 \%) [2].
Surgical excision of the tumor mainly depends on site, size and invasive status of the cancer. Once the lesion is biopsy proven and staged, surgical treatment must focus on complete excision of carcinoma with negative surgical margins, which may be confirmed by intraoperative frozen section [3]. There is no clear evidence as to the required width of negative surgical margins, for a general recommendation, 3-5 $\mathrm{mm}$ can be considered a safe maximum $[4,5]$. Other benefits of surgery are to reduce the pain and bleeding from the lesion itself. Therefore, we recommend early surgery to minimize the risk of disease spread, reducing pain, discomfort and to control bleeding from tumor.

\section{ONCOLOGIST'S PERSPECTIVE}

Malik and Aziz describe the case of a spindle cell tumor (SCS) of the pelvis managed with surgery and a need for re-resection/radiation therapy. The tumor described (spindle cell sarcomas) are a very rare group of tumors where the general principles of sarcoma management are applied. These consist of preoperative radiation therapy, surgery alone, postoperative radiation and chemotherapy. It is no small task to get an acceptable negative surgical margins in the pelvis. In a large study of 6378 patients who underwent preoperative radiation therapy (RT) and surgical resection for a localized extremity or pelvic STS between 2004 to 2014, 23\%; i.e. 1438 , underwent neoadjuvant chemotherapy, and $24 \%$ i.e. 1531 underwent radiation. A total of 2176 patients were included for analysis, with a mean pre-RT-surgery interval of $35 \pm 16$ days. With the numbers available, the study showed that a delay in surgery up to 120 days after radiation is not associated with poorer survival. Therefore, clinicians may be able to delay surgery to minimize the risks of wound complications etc. and not effect outcome [6].

In a SEER data based analysis 3299 SCS cases were identified. White people account for $79.1 \%$ with median age of 57 years without predominance in any gender. A total of 216 cases were pelvic and 206 were abdominal [7]. Within the pelvis these tumors have been seen to arise from the vagina, prostate and other sites [8-12]. Since there are very few cases described individually the role of chemotherapy and radiation can only be extrapolated. An analysis of the US sarcoma collaborative group indicated that RT may play a larger role in minimizing local recurrence than even the margin status. RT may decrease local recurrence to similar rates as a negative margin without RT and may be considered as therapeutic choice with close/positive margins [13]. Whether chemotherapy needs to be given in a high grade tumor such as is presented can only be extrapolated from generalized evidence as the subsets of sarcomas are very small and make it impossible to reach specific conclusions [7]. 
Recent practices tell that any patient with MSI unstable tumor is a candidate for PDI immunotherapy as are those with NTRK mutations with larotrectinib.

\section{REFERENCES}

1. BackeS DM, Kurman RJ, Pimenta JM, Smith JS. Systematic review of human papillomavirus prevalence in invasive penile cancer. Cancer Causes Control 2009; 20: 449-57.

2. Velazquez EF, Melamed J, Barreto JE, Aguero F, Cubilla AL. Sarcomatoid carcinoma of the penis: a clinicopathologic study of 15 cases. Am J Surg Pathol 2005; 29: 1152-8.

3. Leijte JA, Kirrander $P$, Antonini N, Windahl T, Horenblas S. Recurrence patterns of squamous cell carcinoma of the penis: recommendations for follow-up based on a two-centre analysis of 700 patients. Eur Urol 2008; 54: 161-8.

4. Mahesan T, Hegarty PK, Watkin NA. Advances in penile-preserving surgical approaches in the management of penile tumors. Urol Clin North Am 2016; 43: 427-34.

5. Fras AP, Grazio SAF. Hyalinizing spindle cell tumour with giant rosettes of the broad liga-ment. Gynecol Oncol 2001; 83: 405-8.
6. Collier CD, Kim CY, Liu RW, Getty PJ. The interval between preoperative radiation and sur-gery is not associated with overall survival for soft-tissue sarcomas: an analysis of the national cancer database. Clin Orthop Relat Res 2020. [Online ahead of print]

7. Bajpai J, Susan D. Adjuvant chemotherapy in soft tissue sarcomas...Conflicts, consensus, and controversies. South Asian J Cancer 2016; 5: 15-9.

8. Feng L, Wang M, Yibulayin F, et al. Spindle cell sarcoma: a SEER population-based analysis. Sci Rep 2018; 8: 5024.

9. Asaadi M, Najmi J, Carter HW, Bonomo M. Spindle cell sarcoma of scrotum. Urology 1980; 16: 525-6.

10. Strickland P, Perry HC. Spindle cell sarcoma of the vagina; a case treated by radium implant. J Obstet Gynaecol Br Emp 1956; 63 : 280-81.

11. Sarjeant, Hicks, Knight, Harris. Spindle cell sarcoma of the prostate. Can Med Assoc J 1927; 17: 81-4.

12. Afridi SP, Rasool M, Shafiq UR. Spindle cell tumour presenting as an abdominal mass. J Coll Physicians Surg Pak 2010; 20: 687-8.

13. Gannon NP, King DM, Ethun CG, et al. The role of radiation therapy and margin width in localized soft-tissue sarcoma: Analysis from the US sarcoma collaborative. J Surg Oncol 2019; 120: 325-31.

\section{Ans \# 1.}

Ifosfamide induced encephalopathy.

Ans \# 2.

Solid Pseudopapillary Neoplasm. 